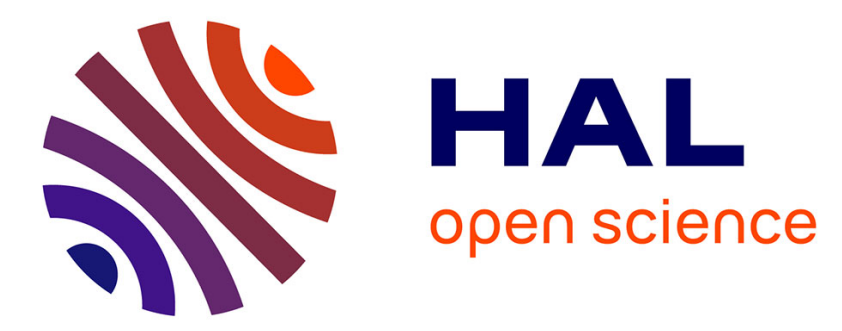

\title{
Réadaptation cardiaque des adultes avec cardiopathies congénitales
}

S. Corone, G. Bosser, A. Legendre, S. Guillaumont, P. Amedro

\section{To cite this version:}

S. Corone, G. Bosser, A. Legendre, S. Guillaumont, P. Amedro. Réadaptation cardiaque des adultes avec cardiopathies congénitales. Annales de Cardiologie et d'Angéiologie, 2018, 10.1016/j.ancard.2018.08.008 . hal-01882607

\section{HAL Id: hal-01882607 \\ https://hal.umontpellier.fr/hal-01882607}

Submitted on 8 Jan 2020

HAL is a multi-disciplinary open access archive for the deposit and dissemination of scientific research documents, whether they are published or not. The documents may come from teaching and research institutions in France or abroad, or from public or private research centers.
L'archive ouverte pluridisciplinaire HAL, est destinée au dépôt et à la diffusion de documents scientifiques de niveau recherche, publiés ou non, émanant des établissements d'enseignement et de recherche français ou étrangers, des laboratoires publics ou privés. 


\title{
Réadaptation cardiaque des adultes avec cardiopathies congénitales
}

\section{Cardiac rehabilitation in adults with congenital heart diseases}

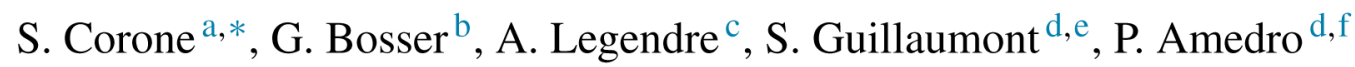 \\ ${ }^{a}$ Service de réadaptation cardiaque, centre hospitalier de Bligny, 91640 Briis-sous-Forges, France \\ ${ }^{\mathrm{b}}$ Service de cardiologie congénitale et pédiatrique, centre de compétences des malformations cardiaques congénitales complexes-M3C, CHRU de Nancy, allée de \\ Morvan, 54500 Vandouvre-lès-Nancy, France \\ ${ }^{c}$ Service de cardiologie pédiatrique, centre de référence des malformations cardiaques congénitales complexes-M3C, hôpital Necker, 75015 Paris, France \\ ${ }^{\mathrm{d}}$ Service de cardiologie pédiatrique et congénitale, centre de compétences des malformations cardiaques congénitales complexes-M3C, CHU de Montpellier, \\ 34090 Montpellier, France \\ ${ }^{\mathrm{e}}$ Unité d'évaluation et de réadaptation en cardiologie pédiatrique, institut-Saint-Pierre, 34250 Palavas-Les-Flots, France \\ ${ }^{\mathrm{f}}$ PHYMEDEXP, CNRS, Inserm, université de Montpellier, 34090 Montpellier, France
}

\begin{abstract}
Résumé
Les grands progrès de la prise en charge médicale et chirurgicale des cardiopathies congénitales ont permis à de nombreux enfants de parvenir à l'âge adulte avec souvent un bon résultat hémodynamique. Mais la plupart de ces adultes ont néanmoins une limitation de leur capacité fonctionnelle. Cette limitation, plus ou moins importante, les pénalise dans leur vie quotidienne et altère leur qualité de vie. L'origine de cette limitation est en générale multifactorielle. Elle est liée, bien sûr, à la sévérité de la cardiopathie et à la qualité du résultat opératoire. Mais il existe très fréquemment un déconditionnement physique. Lui-même peut être secondaire à la cardiopathie mais il est aussi bien souvent secondaire à un manque d'activité physique. Ce sont les parents, parfois surprotecteurs, mais surtout les médecins qui ont imposé, souvent à tort, cette restriction. La réadaptation cardiaque constitue un outil privilégié pour délivrer des conseils, dans un environnement adapté et apporte des bénéfices importants sur la santé et la qualité de vie. Ceci nécessite une collaboration étroite entre les réadaptateurs cardiaques et les cardiologues congénitalistes pour proposer des prises en charge adaptées. Nous apportons ici quelques réflexions et les éléments de base pour guider le réentraînement des ces patients.
\end{abstract}

Mots clés : Réadaptation cardiaque ; Cardiopathie congénitale ; Adultes ; Déconditionnement ; Capacité fonctionnelle ; Activité physique

\begin{abstract}
The great advances in the medical and surgical management of congenital heart diseases have allowed many children to reach adulthood with often a good hemodynamic result. Nevertheless most of these adults have a limitation of their functional capacity. This limitation is more or less important, penalizes them in their daily life and alters their quality of life. The origin of this limitation is generally multifactorial. It is linked, of course, to the severity of the heart disease and the quality of the operative result. But there is very often a physical deconditioning. It can be secondary to the heart disease but is often secondary to a lack of physical activity. It is the parents, sometimes overprotective, but frequently the doctors who imposed, often wrongly, this restriction. It is essential to take this dimension into account in view of the important benefits expected for health and quality of life. Cardiac rehabilitation is a privileged tool for providing advice in a suitable environment. This requires close collaboration between cardiac rehabilitators and congenital cardiologists to offer appropriate care. We bring here some reflections and the basic elements to guide the re-training of these patients.
\end{abstract}

Keywords: Cardiac rehabilitation; Congenital heart disease; Adults; Physical deconditioning; Fitness; Exercise training

\footnotetext{
* Auteur correspondant.

Adressese-mail:s.corone@chbligny.fr, soniacorone@hotmail.com (S. Corone).
} 


\section{Introduction}

Dans les années 1960, $80 \%$ des enfants nés avec une cardiopathie congénitale $(\mathrm{CC})$ décédaient avant d'atteindre l'âge adulte. En 2010, $80 \%$ de ces nourrissons grandissent et deviennent adulte. En 2016, la population des congénitaux adultes dépasse la population pédiatrique et elle ne va que s'accroître au cours des années à venir.

Après avoir concentré les efforts sur la survie de ces enfants, il est indispensable maintenant de s'intéresser à leur qualité de vie et à la prévention secondaire.

Si les sociétés scientifiques cardiologiques européennes [1] et américaines [2] recommandent la pratique d'activités sportives pour la plupart des patients atteints de $\mathrm{CC}$, une attitude restrictive des médecins et surprotectrice des parents a écarté ces enfants des cours d'éducation physique et sportive et des clubs de sport pendant de nombreuses années [3]. Ainsi, ce sont souvent l'appréhension et l'anxiété inappropriées qui rendent difficile la pratique d'une activité physique ou sportive, même en loisir.

Or cette pratique, effectuée de façon régulière, a maintenant bien démontré ses bénéfices en termes de capacité fonctionnelle, de bien-être psychologique et d'intégration sociale [4-6]. À plus long terme, elle a un effet préventif sur la maladie athéromateuse [7] et les pathologies cancéreuses. De plus, commencer jeune à pratiquer une activité physique permet de minimiser le risque de sédentarité en vieillissant.

\section{Qu'est que la réadaptation cardiaque ?}

Un programme de réadaptation cardiaque est centré sur le patient et sa pathologie. Il repose sur un trépied qui comporte des séances d'entraînement à l'effort, une optimisation du traitement médical et un programme d'éducation thérapeutique du patient (ETP). Cette éducation doit être adaptée au type de pathologie et elle doit personnalisée, centrée sur le patient, ce qui nécessite, idéalement, un programme d'ETP spécifique autorisé par les agences régionales de santé dédié aux CC. Mais nous nous concentrerons dans la suite de ce travail principalement sur les spécificités du réentraînement à l'effort des CC qui requiert un abord particulier.

Ce stage peut être effectué en période postopératoire immédiate ou à distance d'une intervention chirurgicale ou encore dans le cadre d'une préparation à la grossesse. Mais il faudra savoir le proposer à tout patient déconditionné afin de lui permettre de débuter et prolonger une activité physique adaptée. Il va permettre de guider les premiers pas du patient dans la pratique d'un entraînement physique adapté, personnalisé, et accompagné d'encouragements.

La pratique d'un exercice physique régulier quotidien en sécurité va rassurer le patient, lui procurer rapidement un gain en souplesse, un certain degré de bien-être après l'effort et un meilleur contrôle pondéral. Secondairement apparaîtront les bénéfices en termes de capacité d'effort maximal et sousmaximal. Le but final étant d'avoir acauis du savoir-faire. du plaisir, de la détente et de la motivation pour poursuivre cette pratique d'exercice physique régulier.

\section{Cardiopathies congénitales et capacité physique}

Les patients porteurs d'une $\mathrm{CC}$ ont un niveau d'activité physique inférieur de 25 à $50 \%$ par rapport à celui de la population générale, générant un sur-risque cardiovasculaire lié à la sédentarité, et donc cumulé avec celui de la CC sous-jacente [8]. La majorité des patients adultes ont ainsi le plus souvent, même pour des CC simples et bien corrigées, telles que les communications interauriculaires, une aptitude physique altérée [9].

Chez les enfants, le niveau d'aptitude physique est dans les limites de la normale pour la plupart des CC, sauf dans les cardiopathies univentriculaires, mais néanmoins significativement plus faible que la population générale [10]. L'origine en est multifactorielle $[9,11,12]$ : perturbations hémodynamiques, anomalies électriques, augmentations de pression et/ou de volume ventriculaire, altérations du système sympathique, augmentation de l'activité neurohormonale, diminution des capacités respiratoires, et modifications du métabolisme musculaire incluant les muscles respiratoires. La persistance d'un shunt droite-gauche avec une cyanose peut également participer à la mauvaise tolérance à l'exercice par diminution de l'apport en oxygène aux muscles périphériques [13-15].

La pratique du sport peut ainsi représenter une difficulté importante pour ces patients. La chirurgie peut également avoir laissé des cicatrices, des adhérences et des douleurs résiduelles rendant difficiles la fréquentation d'une salle de fitness ou d'une piscine. De plus, la prescription d'une activité physique régulière est rarement intégrée à la consultation de suivi [16]. Les raisons sont multiples : manque de formation ou idées reçues de la part des professionnels de santé, des patients ou de leur entourage ; peur vis-à-vis des risques potentiels, notamment celui de la mort subite ; manque d'études spécifiques.

Par conséquence, la force musculaire [17] chez beaucoup de patients porteurs de CC est réduite, comparée aux valeurs de références, que ce soit aux membres supérieurs ou inférieurs. S'installe ainsi, dès l'adolescence, le cercle vicieux du déconditionnement avec fatigue et essoufflement renforçant la sédentarité qui elle même réduit la masse musculaire et engendre plus de fatigue et plus d'essoufflement lors du prochain effort [18].

\section{4. Épreuve d'effort cardiorespiratoire}

Les tests d'effort avec études des échanges respiratoires, et en particulier de la consommation en oxygène $\left(\mathrm{VO}_{2}\right)$, ont un intérêt tout particulier pour l'évaluation des patients atteints de CC même s'ils sont de réalisation plus complexe que les tests d'effort simples [15,19-22].

Plusieurs études réalisées chez des adultes jeunes permettent de mesurer l'impact des différentes CC sur la performance à l'effort $[9,12,23]$. Ces études montrent que, dans leur ensemble, les sujets atteints de CC ont une capacité à l'effort diminuée et nettement inférieure à la normale. Le Tableau 1 indique la 
Tableau 1

Capacité fonctionnelle selon les différentes cardiopathies congénitales exprimée en pourcentage du pic théorique de $\mathrm{VO}_{2}[9,12,23]$.

\begin{tabular}{ll}
\hline & $\%$ du pic théorique de $\mathrm{VO}_{2}$ \\
\hline Coarctation opérée & 78 \\
Valvulopathies opérées & $73-80$ \\
CIA, CIV opérée & $72-75$ \\
Tétralogie de Fallot opérée & $71-72$ \\
Ventricule droit systémique & $62-66$ \\
Anomalie d'Ebstein & $59-60$ \\
Circulation de Fontan & $56-59$ \\
Cardiopathie complexe cyanogène & $42-46$ \\
Syndrome d'Eisenmenger & $39-43$ \\
\hline
\end{tabular}

capacité fonctionnelle selon les différentes $\mathrm{CC}$, exprimée en pourcentage du pic théorique de $\mathrm{VO}_{2}$.

Ces données confirment, sans surprise, la très importante limitation à l'effort des cardiopathies les plus sévères comme les circulations de Fontan, les cardiopathies cyanogènes et l'hypertension artérielle pulmonaire fixée sur shunt (syndrome d'Eisenmenger). Cependant ces études montrent également que l'aptitude aérobie $\left(\mathrm{VO}_{2 \max }\right)$ peut également être altérée dans des cardiopathies simples réparées, telles que les communications interauriculaires ou interventriculaires [24]. Cela reflète à la fois l'existence de séquelles résiduelles et aussi un certain niveau de déconditionnement qui incite à favoriser la pratique de l'activité physique et renforce l'intérêt d'un programme de réadaptation cardiaque.

Le suivi des adultes porteurs de CC par un test d'effort cardiorespiratoire fait désormais partie des recommandations [1]. Cet examen participe à la promotion de l'activité physique et constitue le point de départ des programmes de réadaptation cardiaque.

Enfin, ce test permet aussi d'étudier le $1^{\mathrm{er}}$ seuil ventilatoire (SV1) qui correspond globalement au niveau le plus élevé auquel il est possible de maintenir un effort en endurance aérobie avec une bonne tolérance et une charge cardiovasculaire peu importante. C'est un élément très important pour guider l'entraînement et pour évaluer la tolérance des efforts de la vie quotidienne ou du travail.

\section{5. À quel adulte atteint de cardiopathie congénitale doit-on proposer un programme de réadaptation cardiaque ?}

Dans tous ces cas de CC, même bien réparées par une chirurgie effectuée dans l'enfance, l'intérêt de l'entraînement physique régulier est de lutter contre les risques qui pourraient se cumuler avec la cardiopathie sous-jacente : sédentarité, surpoids, diabète, hypertension artérielle et coronaropathie. Et il y aura aussi un bénéfice considérable sur la qualité de vie [1,2].

Dans le cas des CC complexes, les patients ont subi dès l'enfance une ou plusieurs interventions (cathétérismes et chirurgies cardiaques) et parfois la malformation cardiaque fonctionne grâce à une intervention palliative.

Dans leur vie quotidienne d'adulte, ils sont confrontés aux problèmes de tous les jeunes : trouver du travail, être en forme, faire un emprunt bancaire et mener une grossesse. Ces difficultés sont bien évidement considérablement augmentées du fait de leur pathologie cardiaque. Ces patients doivent pouvoir également bénéficier d'un programme d'éducation thérapeutique qui prend en considération ces aspects, et cela peut tout à fait s'intégrer dans un programme de réadaptation cardiaque [18]. Actuellement, ils sont essentiellement abordés lors des programmes d'ETP sur la transition entre adolescence et âge adulte $[25,26]$.

\section{6. Évaluation des cardiopathies congénitales avant la réadaptation cardiaque}

Il existe une grande diversité de $\mathrm{CC}$ avec des résultats variables en termes de réparation chirurgicale ou par cathétérisme interventionnel. Plusieurs de ces cardiopathies nécessitent des évaluations spécifiques et une collaboration entre cardiologues congénitalistes et l'équipe de cardiologie des centres de réadaptation cardiaque.

Dans leur analyse, Budts W et al. proposent une évaluation de la $\mathrm{CC}$ avant réadaptation sur 5 points : les paramètres ventriculaires (fonction, hypertrophie, dilatation), la pression pulmonaire, la taille de l'aorte, le statut rythmique et l'existence d'une cyanose [27]. Cette méthode simple permet d'orienter vers une activité physique d'intensité faible, modérée ou haute en fonction du niveau de fréquence cardiaque et de l'échelle de Borg [27]. Ensuite, sans être exhaustifs, nous résumons ci-après les spécificités des grands groupes de CC.

\subsection{Cardiopathies avec shunt gauche-droite, opérées}

Globalement, il conviendra de rechercher un shunt résiduel et une hypertension artérielle pulmonaire. Un valvulopathie aortique peut exister en particulier dans les CIV périmembraneuses.

\subsection{Sténoses valvulaire pulmonaire : traitées et non traitées}

La sténose valvulaire pulmonaire est jugée peu sévère lorsque le gradient maximal est $<40 \mathrm{~mm} \mathrm{Hg}$, modérée entre 40 et $60 \mathrm{~mm} \mathrm{Hg}$ et sévère au-delà de $60 \mathrm{~mm} \mathrm{Hg}$.

\subsection{Sténose valvulaire aortique : traitées et non traitées}

L'évaluation des valvulopathies aortiques congénitales ne diffère pas véritablement de celles acquises. Une attention particulière doit être apportée à la dilatation de l'aorte ascendante associée aux bicuspidies [28].

\subsection{Coarctation de l'aorte}

L'évaluation d'une coarctation repose sur l'examen clinique avec mesure de la PA aux membres supérieurs et inférieurs, On dépistera, en particulier, un obstacle résiduel avec gradient tensionnel ( $>20 \mathrm{~mm} \mathrm{Hg}$ ou pas) entre membres supérieurs et inférieurs et une HTA d'effort. 


\subsection{Dysfonction ventriculaire après chirurgie des $C C$}

De nombreux patients conservent une dysfonction ventriculaire précoce ou tardive après la correction de leur CC ce qui, bien sûr, altère leur aptitude à l'effort. L'évaluation échocardiographique de la fonction ventriculaire est plus simple pour les VG en position systémique que pour les VD mais dans ces situations l'IRM (voire la scintigraphie si l'IRM est contre-indiquée) est d'un grand apport. Une dysfonction ventriculaire est jugée sévère si la fraction d'éjection (FE) est inférieure à $40 \%$, modérée si la FE est entre 40 et $50 \%$, et normale si la FE est supérieure à $50 \%$.

\subsection{Cardiopathies cyanogènes}

Ces patients ont tous une limitation plus ou moins sévère à l'effort. Un déficit en fer peut aggraver la tolérance et il est facile à corriger. Chaque situation doit être évaluée sur une base individuelle mais d'une façon générale ces patients devront avoir un réentraînement prudent.

\subsection{Tétralogie de Fallot opérée}

Les enfants avec une tétralogie de Fallot sont habituellement opérés dans la première année de vie. Beaucoup d'entre eux conservent à l'âge adulte une insuffisance pulmonaire significative qui doit être évaluée (protocole de soin HAS 2008 : https:// www.has-sante.fr/portail/upload/docs/application/pdf/2008-10/ guide_pnds_fallot.pdf). L'évaluation de la fonction des deux ventricules est indispensable de même que le test d'effort qui peut permettre de démasquer des arythmies d'effort [29].

\subsection{Transposition des gros vaisseaux corrigée à l'étage atrial}

Les procédures de détransposition à l'étage atrial (Mustard et Senning) ont été réalisées entre les années 1960 et le début des années 1990. Elles ont pour inconvénient majeur de laisser le ventricule droit en position sous-aortique (systémique) et celuici évolue, en général, vers une dysfonction dans la troisième ou quatrième décennie [30]. Compte tenu du remaniement atrial les troubles du rythme supraventriculaires sont fréquents.

\subsection{Transposition congénitalement corrigée des gros vaisseaux (TCCGV), double discordance}

Cette cardiopathie, dans laquelle le ventricule droit est en position systémique, comporte souvent des lésions associées dont les plus fréquentes sont une CIV et une sténose souspulmonaire et valvulaire pulmonaire. La tolérance à l'effort est souvent altérée [30] et la capacité d'effort ainsi que la dysfonction du VD systémique sont des facteurs prédictifs de complications. Il est important d'évaluer aussi la fonction de la valve atrioventriculaire systémique et les obstacles résiduels sur les voies d'éjection ventriculaires.

\subsection{Transposition des gros vaisseaux corrigée à l'étage artériel (switch)}

Cette chirurgie, qui a de très bons résultats, est devenue la règle depuis une trentaine d'année. Il convient d'évaluer particulièrement les sténoses coronaires résiduelles, les sténoses résiduelles sur la voie pulmonaire qui sont, fort heureusement, souvent peu sévères et la fuite aortique (ancienne valve pulmonaire).

\subsection{Anastomose cavo-pulmonaires (procédures de Fontan)}

Ces interventions palliatives, réalisées en cas de ventricule unique, consistent en une redirection complète du sang veineux vers les artères pulmonaires. Les patients ont en général une très importante altération de leur capacité à l'effort et cette capacité est fortement corrélée à l'espérance de vie [29,31]. Cette limitation est multifactorielle et les patients doivent bénéficier d'une évaluation complète, des lésions associées et de la fonction du ventricule unique par imagerie. Il existe une insuffisance chronotrope à l'effort parfois marquée dans les circulations de Fontan.

\subsection{L'hypertension pulmonaire}

Il est important de signaler que l'hypertension artérielle pulmonaire n'est plus considérée comme une contre indication à la réadaptation cardiaque. Depuis 10 ans, l'équipe allemande de Heidelberg a publié plusieurs études sur des patients avec une hypertension pulmonaire sévère (pression moyenne $60 \mathrm{mmHg}$ ) et des traitements lourds. Des résultats très encourageants ont été obtenus avec des programmes comportant de l'exercice en centre pendant 3 semaines à raison de $1 \mathrm{~h} 30$ par jour (vélo, renforcement musculaire et marche) puis à domicile pendant 12 semaines [32].

\section{Pratique et spécificités du réentrânement des cardiopathies congénitales}

Comme nous l'avons signalé précédemment, il y a toujours un intérêt à la pratique régulière d'une activité physique modérée favorisant, selon les recommandations internationales, l'endurance aérobie c'est-à-dire au SV1 $[19,27]$.

Avant de prescrire l'activité physique, 4 étapes sont indispensables :

- faire le bilan de la cardiopathie et de son équilibre hémodynamique au repos. Une collaboration étroite avec le cardiologue congénitaliste est recommandée. Rechercher les contre-indications éventuelles ;

- évaluer la capacité d'adaptation à l'effort du patient grâce à une épreuve d'effort cardiorespiratoire sur tapis ou sur vélo avec incrémentation progressive de la charge en rampe tenant compte du niveau de déconditionnement ; 


\section{PERCEPTION DE L'EFFORT}

ECHELLE DE BORG (6 - 20)

\section{Pas d'effort \\ 7 \\ 8 \\ Extrêmement léger \\ 9 Très léger}

10

11

Léger

12

13

Relativement difficile

14

15

Difficile

16

\section{Très difficile}

18

\section{Extrêmement difficile}

\section{Effort maximal}

Fig. 1. Échelle de Borg 6-20.

- déterminer le ou les facteurs limitant(s) de la capacité d'effort : fonction respiratoire, cardiopathie, muscles ou bien encore le psychisme ? Volonté ou appréhension?

- déterminer les paramètres permettant de guider la prescription et les conseils. Fc et $\mathrm{VO}_{2}$ au pic et au SV1.

La prescription de l'activité physique associera : endurance aérobie au SV1 (cf. supra), renforcement musculaire et gymnastique douce comportant stretching et exercices de coordination.

Dans les CC les plus complexes, le déconditionnement peut être marqué et l'intensité de démarrage de l'effort se situera alors à un niveau faible. Si on ne dispose pas des résultats de l'analyse de $\mathrm{VO}_{2}$, mais surtout pour guider la progression de l'entraînement, on peut utiliser deux méthodes simples pour donner des consignes de travail au SV1 : d'une part, ce que les anglo-saxons appellent le «talk-test » et, d'autre part, les échelles de perception fonctionnelles de la pénibilité de l'effort comme l'échelle de Borg 6-20 (Fig. 1).

Pour le «talk-test » le principe est de régler le niveau d'effort sur le niveau d'essoufflement en ne dépassant pas l'aisance respiratoire et un niveau ou on peut parler mais pas chanter ( « talk but not sing »). L'échelle de Borg va de 6, correspondant au repos, à 20, correspondant à l'effort maximal. Et elle est centrée sur le niveau 13 correspondant par définition à un effort modéré mais soutenu que l'on est susceptible de poursuivre en endurance, ce qui correspondant justement au SV1.
Pour nous, généralement, il est prudent de débuter quelques séances d'entraînement à un niveau de 11-12 sur l'échelle de Borg. Par la suite, avec la progression, on vise un niveau 13 à 14 sur cette même échelle.

L'entraînement en fractionné, plus ludique, peut être proposé selon différentes modalités [18,33]. Les protocoles avec hautes intensités doivent être réservés aux situations les plus favorables. Une des possibilités est d'utiliser le mode $40 \% / 80 \%$ de la charge maximale atteinte au test d'effort avec des paliers de 3 minutes pour la charge basse et 1 minute pour le palier haut. L'augmentation en commençant par le palier du bas peut être intéressante. La progression doit être particulièrement douce chez ces patients.

En centre, le vélo, la marche sur tapis ou en extérieur sont les activités d'endurance le plus souvent proposés. La marche est particulièrement importante pour augmenter le retour veineux. Elle est à privilégier chez les patients avec circulation de Fontan et les dérivations cavo-pulmonaire totales.

De l'oxygène nasal est rarement utilisé. Cependant, il peut permettre de passer un cap ou de travailler à une charge un peu plus intense et d'améliorer le confort de certains patients.

La kinésithérapie respiratoire, le lancer de sarbacane sur une cible et les vocalises sont des activités qui permettent d'augmenter les volumes ventilatoires (inspiratoire et expiratoire) et sont toujours associés.

Il paraît intéressant de diversifier les activités et de faire découvrir d'autres activités physiques telles que la marche nordique, le Tai chi, le Yoga, la gymnastique Pilate, la natation, la marche en milieu aquatique, le tir à l'arc, etc.

En fin de séjour, grâce à un entretien motivationnel, des objectifs réalistes seront établis en essayant de définir le type d'activité et leur rythme avec précisions et en les inscrivant dans l'agenda de la personne. Un éventuel programme de rappel à 6 mois ou 1 an est toujours proposé.

Des programmes de réadaptation cardiovasculaire avec relais à domicile et favorisant l'ambulatoire et la supervision sont conseillés par les experts et considérés sans risque supplémentaire tout en améliorant la motivation et l'adhésion [34]. Les auteurs ayant rapporté une expérience chez les patients porteurs de $\mathrm{CC}$, retiennent ce type de programme que ce soit chez l'enfant ou le jeune adulte [18].

\section{Résultats de la réadaptation dans la littérature et selon type de $\mathrm{CC}$}

Les effets bénéfiques de la réadaptation cardiaque sur le plan fonctionnel mais aussi en termes de qualité de vie chez les adultes porteurs de CC sont de plus en plus étudiés [8,12,35-38]. Dans la revue de la littérature très complète de Duppen et al., un gain moyen de $2,4 \mathrm{~mL} / \mathrm{min} / \mathrm{kg}$ de pic de $\mathrm{VO}_{2}$ a été mesuré chez 177 adultes atteints de CC participant à un programme d'entraînement [39]. Cette augmentation serait majorée à $3,6 \mathrm{~mL} / \mathrm{min} / \mathrm{kg}$ selon Gomes-Neto et al. pour les études pédiatriques soulignant l'intérêt à débuter ce type de programme dés l'adolescence [38].

Chez des jeunes hollandais avec une tétralogie de Fallot ou une circulation de Fontan, la même équipe a montré que le 
pic de $\mathrm{VO}_{2}$ augmentait en moyenne de $5 \%$ par rapport au groupe témoin, après un programme de 12 semaines comportant 3 heures d'exercice en aérobie par semaine à 60-70\% de la fréquence cardiaque de réserve [29].

Dans leur méta-analyse regroupant 200 participants porteurs d'un ventricule unique avec circulation de Fontan, Sutherland et al. ont montré l'absence de danger des programmes de réadaptation cardiaque avec travail musculaire en endurance aérobie, associés à un travail musculaire en résistance [40]. Les exercices en résistance des membres inférieurs permettent d'augmenter le retour veineux et la précharge par effet de chasse de la pompe musculaire périphérique. Volume d'éjection, débit cardiaque et capacité d'effort sont ainsi améliorés. Cette méta-analyse évoque un bénéfice important sur la tolérance à l'effort, la force musculaire et la qualité de vie, chez des patients dont l'aptitude aérobie est particulièrement altérée.

Chez les patients avec un ventricule droit en position systémique, Winter et al. ont mesuré un gain en pic de VO2 de $3,4 \mathrm{~mL} / \mathrm{min} / \mathrm{kg}(+7 \%)$ chez 24 patients ayant participé à un 10 semaines de réentraînement par rapport aux patients contrôles [41].

Enfin, plusieurs études ont démontré, d'une part, qu'il existe une forte corrélation entre la qualité de vie et la $\mathrm{VO}_{2}$ chez les patients atteints de $\mathrm{CC}$ [5] et, d'autre part, que la mauvaise capacité d'effort et le faible pic de $\mathrm{VO}_{2}$ sont des éléments de mauvais pronostic $[9,42,43]$. En améliorant ce pic de $\mathrm{VO}_{2}$ par la réadaptation, on peut donc favoriser une amélioration du pronostic chez ces patients, même si des études le démontrant font encore défaut pour cette population qui accède peu à la réadaptation cardiaque.

\section{Sécurité de la réadaptation cardiaque dans les cardiopathies congénitales}

Même si elles sont encore limitées, les données de la littérature concernant la réadaptation cardiaque chez les patients porteurs de $\mathrm{CC}$ ne rapportent pas de sur-risque, quel que soit le programme envisagé $[44,45]$. Dans les essais comportant des patients avec hypertension artérielle pulmonaire, il n'y pas non plus eu de complication grave.

L'augmentation importante du volume d'activité physique ne semble pas entraîner une augmentation de la mortalité. Par contre, l'activité intense pratiquée de façon rare peut s'accompagner d'une augmentation transitoire de complications cardiaques.

Ainsi donc, il est indispensable d'apprécier de façon individuelle le risque pour guider le réentraînement à l'effort et de quantifier la balance bénéfices/risques [44,45].

\section{Conclusion}

Les progrès de la cardiologie congénitale sur les 30 dernières années ont amélioré considérablement le pronostic des patients et modifié l'épidémiologie de cette sur-spécialité. Aujourd'hui, de nouvelles stratégies s'offrent aux patients porteurs de CC, telles que la réadaptation cardiaque. L'épreuve d'effort cardiorespiratoire en est le point de départ. Comme chez l'insuffisant cardiaque chronique, il est désormais démontré que l'aptitude aérobie est corrélée à la qualité de vie des patients ayant une CC. Aussi, les principes de la réadaptation de l'insuffisant cardiaque sont-ils applicables aux congénitaux cardiaques, avec quelques spécificités en termes d'hémodynamique, mais aussi de profil psychosocial des patients. Bien que les données de la littérature soient encore assez limitées, les bénéfices de tels programmes de réadaptation cardiaque sont prometteurs chez les adultes porteurs de $\mathrm{CC}$.

Le rôle du cardiologue dans la prescription d'une réadaptation cardiaque est fondamental chez ces patients. En collaborant étroitement avec l'équipe de réadaptation cardiaque et le cardiologue congénitaliste, il peut participer à la promotion de l'activité physique chez ces patients trop longtemps mis sur «le banc de touche ».

Ainsi, les adultes porteurs de CC pourront bénéficier des effets favorables de la réadaptation cardiaque, sur la capacité musculaire, la tolérance fonctionnelle à l'effort, la qualité de vie et à terme, probablement aussi sur la morbi-mortalité.

\section{Déclaration de liens d'intérêts}

Les auteurs déclarent ne pas avoir de liens d'intérêts.

\section{Références}

[1] Baumgartner H, Bonhoeffer P, De Groot NMS, de Haan F, Deanfield JE, Galie N, et al. ESC Guidelines for the management of grown-up congenital heart disease (new version 2010): the Task Force on the management of grown-up congenital heart disease of the European Society of Cardiology (ESC). Endorsed by the Association for European Paediatric Cardiology (AEPC). Eur Heart J 2010;31:2915-57.

[2] Warnes CA, Williams RG, Bashore TM, Child JS, Connolly HM, Dearani JA, et al. ACC/AHA 2008 guidelines for the management of adults with congenital heart disease: a report of the American College of Cardiology/American Heart Association Task Force on Practice Guidelines (Writing Committee to Develop Guidelines on the Management of Adults With Congenital Heart Disease). Developed in Collaboration With the American Society of Echocardiography, Heart Rhythm Society, International Society for Adult Congenital Heart Disease, Society for Cardiovascular Angiography and Interventions, and Society of Thoracic Surgeons. J Am Coll Cardiol 2008;52:e143-263.

[3] Van Hare GF, Ackerman MJ, Evangelista JK, Kovacs RJ, Myerburg RJ, Shafer KM, et al. Eligibility and disqualification recommendations for competitive athletes with cardiovascular abnormalities: Task Force 4: congenital heart disease: a scientific statement from the American Heart Association and American College of Cardiology. Circulation 2015;132:e281-91.

[4] Opotowsky AR, Rhodes J, Landzberg MJ, Bhatt AB, Shafer KM, Yeh DD, et al. A randomized trial comparing cardiac rehabilitation to standard of care for adults with congenital heart disease. World J Pediatr Congenit Heart Surg 2018;9:185-93.

[5] Amedro P, Picot MC, Moniotte S, Dorka R, Bertet H, Guillaumont S, et al. Correlation between cardio-pulmonary exercise test variables and healthrelated quality of life among children with congenital heart diseases. Int $\mathbf{J}$ Cardiol 2016;203:1052-60.

[6] Longmuir PE, Brothers JA, de Ferranti SD, Hayman LL, Van Hare GF, Matherne GP, et al. Promotion of physical activity for children and adults with congenital heart disease: a scientific statement from the American Heart Association. Circulation 2013;127:2147-59.

[7] Engelings CC, Helm PC, Abdul-Khaliq H, Asfour B, Bauer UMM, Baumgartner $\mathrm{H}$, et al. Cause of death in adults with congenital heart disease - An analysis of the German National Register for Congenital Heart Defects. Int J Cardiol 2016;211:31-6. 
[8] Dua JS, Cooper AR, Fox KR, Stuart AG. Physical activity levels in adults with congenital heart disease. Eur J Cardiovasc Prev Rehabil 2007;14:287-93.

[9] Kempny A, Dimopoulos K, Uebing A, Moceri P, Swan L, Gatzoulis MA, et al. Reference values for exercise limitations among adults with congenital heart disease. Relation to activities of daily life - single centre experience and review of published data. Eur Heart J 2012;33:1386-96.

[10] Amedro P, Gavotto A, Guillaumont S, Bertet H, Vincenti M, De La Villeon $\mathrm{G}$, et al. Cardiopulmonary fitness in children with congenital heart diseases versus healthy children. Heart Br Card Soc 2018;104:1026-36.

[11] Trojnarska O, Gwizdała A, Katarzyński S, Katarzyńska A, Szyszka A, Lanocha M, et al. Evaluation of exercise capacity with cardiopulmonary exercise test and B-type natriuretic peptide in adults with congenital heart disease. Cardiol J 2009;16:133-41.

[12] Buys R, Cornelissen V, De Bruaene AV, Stevens A, Coeckelberghs E, Onkelinx $S$, et al. Measures of exercise capacity in adults with congenital heart disease. Int J Cardiol 2011;153:26-30.

[13] Null A. Pathophysiology of pediatric heart failure. Prog Pediatr Cardiol 2000;11:175-84.

[14] Adatia I, Kemp GJ, Taylor DJ, Radda GK, Rajagopalan B, Haworth SG. Abnormalities in skeletal muscle metabolism in cyanotic patients with congenital heart disease: a 31P nuclear magnetic resonance spectroscopy study. Clin Sci Lond Engl 1979 1993;85:105-9.

[15] Feltez G, Coronel CC, Pellanda LC, Lukrafka JL. Exercise capacity in children and adolescents with corrected congenital heart disease. Pediatr Cardiol 2015;36:1075-82.

[16] Swan L, Hillis WS. Exercise prescription in adults with congenital heart disease: a long way to go. Heart Br Card Soc 2000;83:685-7.

[17] Kröönström LA, Johansson L, Zetterström A-K, Dellborg M, Eriksson P, Cider $\AA$. Muscle function in adults with congenital heart disease. Int J Cardiol 2014;170:358-63.

[18] Amedro P, Gavotto A, Bredy C, Guillaumont S. Réadaptation cardiaque de l'enfant et l'adulte avec une cardiopathie congénitale. Presse Med 2017:46:530-7.

[19] Takken T, Giardini A, Reybrouck T, Gewillig M, Hövels-Gürich HH, Longmuir PE, et al. Recommendations for physical activity, recreation sport, and exercise training in paediatric patients with congenital heart disease: a report from the Exercise, Basic \& Translational Research Section of the European Association of Cardiovascular Prevention and Rehabilitation, the European Congenital Heart and Lung Exercise Group, and the Association for European Paediatric Cardiology. Eur J Prev Cardiol 2012;19:1034-65.

[20] Guazzi M, Adams V, Conraads V, Halle M, Mezzani A, Vanhees L, et al. Clinical recommendations for cardiopulmonary exercise testing data assessment in specific patient populations. Eur Heart J 2012;33:2917-27.

[21] Ten Harkel ADJ, Takken T. Exercise testing and prescription in patients with congenital heart disease. Int J Pediatr 2010:2010.

[22] Rhodes J, Ubeda Tikkanen A, Jenkins KJ. Exercise testing and training in children with congenital heart disease. Circulation 2010;122:1957-67.

[23] Inuzuka R, Diller G-P, Borgia F, Benson L, Tay ELW, Alonso-Gonzalez $\mathrm{R}$, et al. Comprehensive use of cardiopulmonary exercise testing identifies adults with congenital heart disease at increased mortality risk in the medium term. Circulation 2012;125:250-9.

[24] Heiberg J, Petersen AK, Laustsen S, Hjortdal VE. Abnormal ventilatory response to exercise in young adults operated for ventricular septal defect in early childhood: a long-term follow-up. Int J Cardiol 2015;194:2-6.

[25] Sable C, Foster E, Uzark K, Bjornsen K, Canobbio MM, Connolly HM, et al. Best practices in managing transition to adulthood for adolescents with congenital heart disease: the transition process and medical and psychosocial issues: a scientific statement from the American Heart Association. Circulation 2011;123:1454-85.

[26] Ladouceur M, Calderon J, Traore M, Cheurfi R, Pagnon C, Khraiche D, et al. Educational needs of adolescents with congenital heart disease: impact of a transition intervention programme. Arch Cardiovasc Dis 2017;110:317-24.
[27] Budts W, Börjesson M, Chessa M, van Buuren F, Trigo Trindade P, Corrado D, et al. Physical activity in adolescents and adults with congenital heart defects: individualized exercise prescription. Eur Heart J 2013;34:3669-74.

[28] Bonow RO, Nishimura RA, Thompson PD, Udelson JE. Valvular heart disease: a scientific statement from the American Heart Association and American College of Cardiology. Circulation 2015;132:e292-7.

[29] Duppen N, Etnel JR, Spaans L, Takken T, van den Berg-Emons RJ, Boersma E, et al. Does exercise training improve cardiopulmonary fitness and daily physical activity in children and young adults with corrected tetralogy of Fallot or Fontan circulation? A randomized controlled trial. Am Heart J 2015;170:606-14.

[30] van der Bom T, Winter MM, Knaake JL, Cervi E, de Vries LSC, Balducci A, et al. Long-term benefits of exercise training in patients with a systemic right ventricle. Int J Cardiol 2015;179:105-11.

[31] Wittekind S, Mays W, Gerdes Y, Knecht S, Hambrook J, Border W, et al. A Novel mechanism for improved exercise performance in pediatric fontan patients after cardiac rehabilitation. Pediatr Cardiol 2018;39:1023-30.

[32] Grünig E, Lichtblau M, Ehlken N, Ghofrani HA, Reichenberger F, Staehler G, et al. Safety and efficacy of exercise training in various forms of pulmonary hypertension. Eur Respir J 2012;40:84-92.

[33] Novaković M, Prokšelj K, Rajkovič U, Vižintin Cuderman T, Janša Trontelj K, Fras Z, et al. Exercise training in adults with repaired tetralogy of Fallot: a randomized controlled pilot study of continuous versus interval training. Int J Cardiol 2018;255:37-44.

[34] Anderson L, Sharp GA, Norton RJ, Dalal H, Dean SG, Jolly K, et al. Homebased versus centre-based cardiac rehabilitation. Cochrane Database Syst Rev 2017;6:CD007130.

[35] Gierat-Haponiuk K, Haponiuk I, Szalewska D, Chojnicki M, Jaworski R, Niedoszytko P, et al. Effect of complex cardiac rehabilitation on physical activity and quality of life during long-term follow-up after surgical correction of congenital heart disease. Kardiol Pol 2015;73:267-73.

[36] Dulfer K, Duppen N, Kuipers IM, Schokking M, van Domburg RT, Verhulst FC, et al. Aerobic exercise influences quality of life of children and youngsters with congenital heart disease: a randomized controlled trial. J Adolesc Health 2014;55:65-72.

[37] Morrison ML, Sands AJ, McCusker CG, McKeown PP, McMahon M, Gordon J, et al. Exercise training improves activity in adolescents with congenital heart disease. Heart Br Card Soc 2013;99:1122-8.

[38] Gomes-Neto M, Saquetto MB, da Silva e Silva CM, Conceição CS, Carvalho VO. Impact of exercise training in aerobic capacity and pulmonary function in children and adolescents after congenital heart disease surgery: a systematic review with meta-analysis. Pediatr Cardiol 2016;37:217-24.

[39] Duppen N, Takken T, Hopman MTE, ten Harkel ADJ, Dulfer K, Utens EMWJ, et al. Systematic review of the effects of physical exercise training programmes in children and young adults with congenital heart disease. Int J Cardiol 2013;168:1779-87.

[40] Sutherland N, Jones B, d'Udekem Y. Should we recommend exercise after the Fontan procedure? Heart Lung Circ 2015;24:753-68.

[41] Winter MM, van der Bom T, de Vries LCS, Balducci A, Bouma BJ, Pieper PG, et al. Exercise training improves exercise capacity in adult patients with a systemic right ventricle: a randomized clinical trial. Eur Heart J 2012;33:1378-85.

[42] Tikkanen AU, Oyaga AR, Riaño OA, Álvaro EM, Rhodes J. Paediatric cardiac rehabilitation in congenital heart disease: a systematic review. Cardiol Young 2012;22:241-50.

[43] Ubeda Tikkanen A, Nathan M, Sleeper LA, Flavin M, Lewis A, Nimec D, et al. Predictors of postoperative rehabilitation therapy following congenital heart surgery. J Am Heart Assoc 2018;7:e008094.

[44] Tutarel O, Gabriel H, Diller G-P. Exercise: friend or foe in adult congenital heart disease? Curr Cardiol Rep 2013;15:416.

[45] Chaix M-A, Marcotte F, Dore A, Mongeon F-P, Mondésert B, Mercier L-A, et al. Risks and benefits of exercise training in adults with congenital heart disease. Can J Cardiol 2016;32:459-66. 